\title{
44th ANNUAL SASKATCHEWAN CHRISTMAS BIRD COUNT - 1985
}

Compiled by MARY I. HOUSTON, 863 University Drive, Saskatoon, Saskatchewan. S7N 0J8

Seventy-five bird counts were received this year - the greatest number ever, seven more than the previous high of 68 in 1984.

Most important are the continuing regular count reports which give a longterm picture of the winter bird life in these areas. It is also gratifying to see counts from new areas, and we certainly hope they will be repeated.

Commendable cooperation by a group feeding birds at and near Kamsack, coordinated by Isabel Ritchie, produced a list of 1068 individuals of 17 species.

The only new species added to the all- time list for Count Day, was a Great Blue Heron seen at Fort Walsh. We can usually count on something different from this area! A new species was added to the Additional List - a Field Sparrow seen at Saskatoon. A red phase Eastern ScreechOwl on the Yorkton count replaces the unidentified screech-owl on the full list. It is confirmed by a (later) photograph taken by Warren Hjertaas. Last year's screech-owl seen at Fort Walsh was unidentified as to species. The Fox Sparrow, reported at Moose Jaw on Count Day, changed its status on the all-time list from Additional to Count Day category. This brings the Count Day total to 141, with 7 Additional Species seen during Count Period but not on Count Day. In

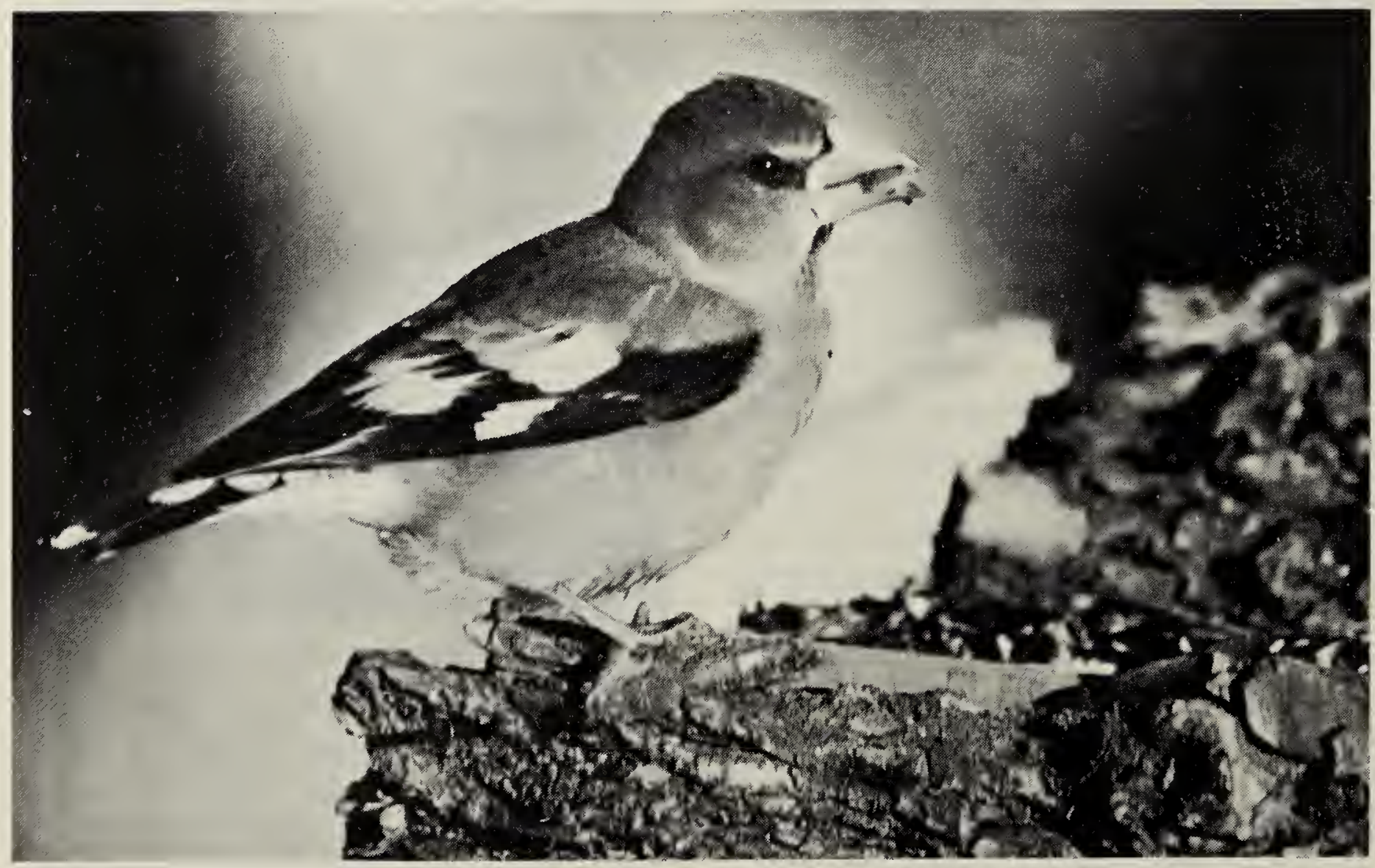


1985, 71 species were seen on Count Day, with 11 additional species reported during the period for a total of 82 . The record high was 84 in 1981. This year's total is second highest.

Black-billed Magpies were reported most frequently (71 localities). Pine Grosbeaks (48), Evening Grosbeaks (45), Hairy Woodpeckers (52) and Downy Woodpeckers (49) were up in number of localities cited. On the other hand Gray Partridges (36) and Snowy Owls (28) were noticeably down. Rosy Finches were seen in two localities only, and in neither case on the day of the count. Confusing finches (Purple? House?) were reported from Endeavour. An incredible 3043 Horned Larks were seen at Govenlock, and although reported on about the usual number of counts, were nowhere else in that quantity.

Two reporters from southern Saskatchewan remarked on the decrease in the population of small birds, particularly House Sparrows, and wondered whether this was an after effect of grasshopper spraying.

Squaw Rapids' 34 species was the highest for Count Day. Fort Walsh and Saskatoon tied for second with 32 each. Fort Walsh, however, added six extra species during count period, while Saskatoon added only two.

After the list of participants from the 75 localities, and the map, Table 1 gives the Coverage and the Weather; Table 2 gives species reported from more than three locations and gives numbers of each species seen in each locality on Count Day, with numbers of additional species during Count Period indicated with a + sign. Table 3 gives numbers and locations of species seen in three or fewer locations. Table 4 gives numbers and locations of the birds unidentified as to species.

\section{Count Areas and Participants}

Names of compilers in italics

1. ARDATH-OUTLOOK. Alan R. Smith.

2. ASSINIBOIA. Ed Bearss, Gordon Brunt, John G. Burgeson, A. Duxbury, Gordon Forsythe, Cecil T. Hayward, Wilf Prentice, Lorne Yeo.

3. BANGOR. George Hilton, Jean Hilton.

4. BIGGAR. Roger Assailly, Don Renaud, Wayne Renaud, Robert Wapple, Guy Wapple, Gordon Webber.

5. BIG GULLY CREEK. Wayne Harris, Sheila Lamont.

6. BIRCH HILLS. Moe Maraschel, Don Weidl.

7. BRIGHTWATER RESERVOIR. Dan Neves, Alan R. Smith.

8. BROADVIEW. Dave J. Chaskavich.

9. BROMHEAD. Carol Bjorklund, Lorne Bjorklund.

10. CLEMENCEAU. Donald F. Hooper.

11. CROOKED LAKE. Don Weidl, Tony Weidl.

12. DALMENY. Loyd Sperling.

13. DILKE. Margaret Belcher.

14. DUCK MOUNTAIN. Dale Hjertaas, Paule Hjertaas.

15. DUVAL. Wayne Harris, Laurel Hendry, Neal Hendry, Sheila Lamont, lain Richardson, Lloyd S. Saul, Victor Tucker.

16. EASTEND. Henri Lebastard.

17. ENDEAVOUR. Norman Harris.

18. FIR RIVER ROAD. Donald $F$. Hooper.

19. FORT QU'APPELLE. Del Cairns, Russ Cairns, Ethel Cockwill, Peggy Cole, Bernard DeVries, Doreen Evans, Doug Evans, Selma Hiebert, Ronald Hooper, Alice Laing, Lois Lamontagne, Don McDougal, Allan Mlazgar, Jean Mlazgar, Raymond Mlazgar, Enola Rak, Doreen Rowell, Lorne Rowell, Bill Smith, Eileen Smith, Dorothy Williams.

20. FORT WALSH. Wayne Harris, Sheila Lamont, Wilkes Parsonage, Guy Wapple, Robert Wapple. 
21. GARDINER DAM. Wayne Harris, Sheila Lamont, Guy Wapple, Robert Wapple.

22. GLAMIS-WISETON. Grev L. Jones.

23. GOODSOIL. Catherine Morton, David Morton, Richard Morton.

24. GOOD SPIRIT LAKE. Bill Anaka, Joyce Anaka.

25. GOVENLOCK. Wayne Harris, Sheila Lamont, Guy Wapple, Robert Wapple.

26. GRAND CENTRE-PIERCELAND. Bill Kirwin, Frank Roy, Ryan Roy.

27. GUERNSEY. Muriel Carlson.

28. HERBERT. Terry Toews, Don Weidl.

29. HUDSON BAY. R. Beaulieu, Albert Bennett, Joyce Hayes, Les Hayes, Donald Hooper.

30. HUMBOLDT. Ed Brockmeyer, Dwayne Saretsky.

31. INDIAN HEAD. Cec and Betty Ashmore, Peter and Isobel Barrett, Coral and Margot Beaulieu, Vic Beaulieu, Hazel Buglass, Eric, Mavis and Roger Gray, Jim and Joan Halford, Don Hayward, Linda Hill, Gordon Howe, Betty and Roy Mclver, Rose and Roy McLauglin, Dora Nichols, Doris Pals, Steven, Tammy and Tim Reihl, Joan and Lorne Scott, Gretie and Reg Scott, Fred Skinner, Joyce Skinner, Mary Skinner, Chas. and Ruby Thompson, Gordon and Ann Willerth, Garth and Barbara Willoughby.

32. KAMSACK. Mable Buceuk, Fred Erhart, Metro Rosowsky, Penny Rezansoff, Isabel Ritchie, Irene Shindler, Tannis Strokoff.

33. KENASTON. JoAnn Beckie, Lawrence Beckie, Lori Beckie, Margaret Beckie.

34. KINDERSLEY. Jean Harris, Tim Harris.

35. KINDERSLEY. Gerry Essar, Michael Essar.

36. KUTAWAGAN LAKE. Wayne Harris, Lloyd Saul.

37. LAKE DIEFENBAKER (Qu'Appelle Dam). Glenn O. Honig.

38. LA RONGE. Jim Paul.
39. LAST MOUNTAIN LAKE. Wayne Harris, Sheila Lamont.

40. LEADER. Daisy D. Meyers.

41. LITTLE BEAR LAKE. Bob Ackerman, Julie Jensen, Ron Jensen.

42. LIVELONG. Sarah Pavka.

43. LOON LAKE. Alf Davis, Frank Scott, Penny Scott.

44. LOVE-TORCH RIVER. Bert Dalziel, Duke Dalziel, Joan Dalziel, Ed Dixon, Betty Donovan, Bruce Donovan, Pearl French, Mildred Long, Bill Matthews, Lynn Matthews, Allen Tatlow.

45. LUSELAND. Kim Finley, Estelle Martfeld, Travis Martfeld.

46. MAIDSTONE BRIDGE. Wayne Harris, Sheila Lamont, Tom Lamont.

47. MARSDEN. Florence Chase.

48. MELFORT. Bud Allen, Evelyn Allen, Genevieve Belliveau, Meghan Curry, Philip Curry, Glenn Galloway, Frieda Markland.

49. MOOSE JAW. Brodie and Mac Aldred, Edith Bell, John Docherty, Carl Ellis, Doug Francis, Alan, Arthur, Edward, Hartley and Lawrence Fredeen, Edith, John and Pat Kern, Cy

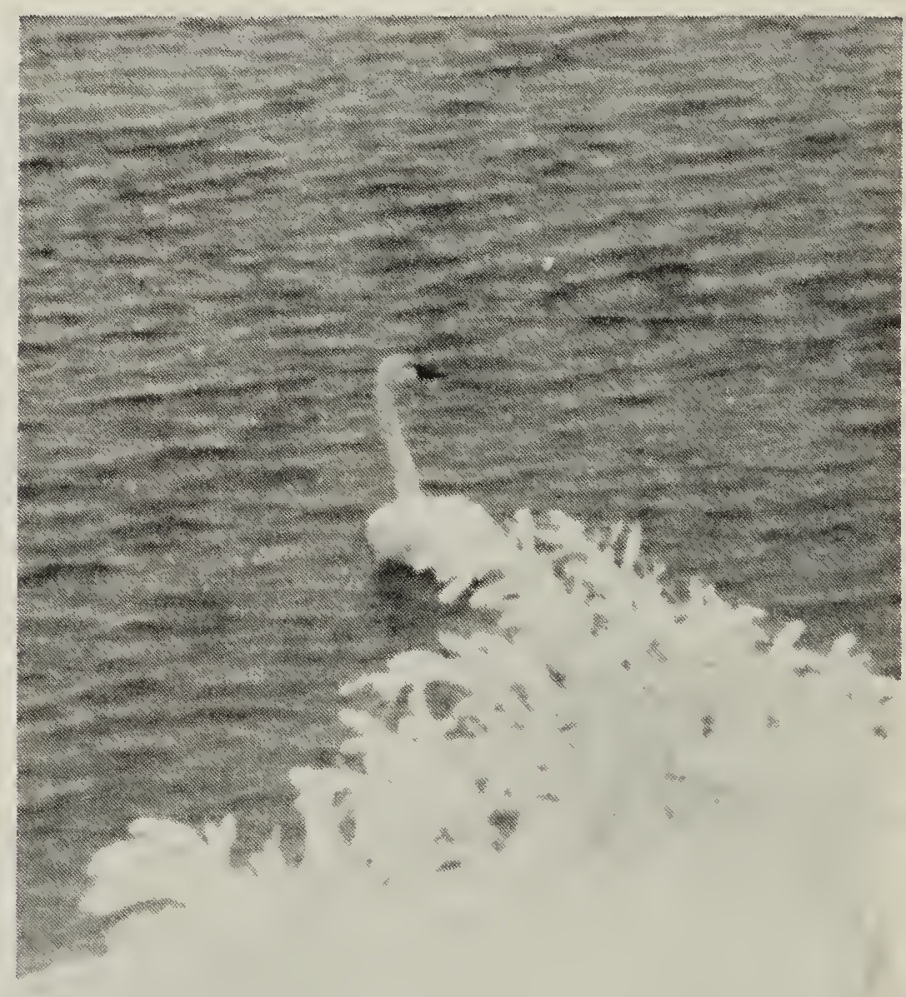

Trumpeter Swan at Squaw Rapids powerchannel 24 December 1986

Wayne C. Harris 
Knight, Leith Knight, Connie and Hugh McIntyre, Doug Neil, Darryl Owens, Brean Peele, Richard and Wilma Pickering, Dave Pyle, Gavina Reekie, Arie and Henry Van Dorland, Sheina Wait.

50. NAICAM. Alan Jensen, Lavawn Jensen, Ron Jensen.

51. NIPAWIN. Bert Dalziel, Bruce Donovan.

52. NIPAWIN. Inge Christiansen, Joyce Christiansen, Ruth Christiansen.

53. PIKE LAKE. Benji, Chris and Jessica Escott, Mary Gilliland, Bob Godwin, Bernie Gollop, Kathy Meeres, Stan Shadick, Charlie Simpson, Jim Wedgwood, Lois Wooding.

54. PINE CREE REGIONAL PARK. Christopher Donovan, James Donovan.

55. PRINCE ALBERT. John Burt, Pam Burt.

56. PRINCE ALBERT NATIONAL PARK. Nancy Alexander, Doug and Marilyn Anions, Susan Carr, Tim Haughian, Ann Landry, Bradley Muir, Derek Petersen, Josee Poirier, Marlis Pollock, Debra Stacey, Merv Syroteuk.

57. RAYMORE. Wayne Harris, Sheila Lamont.

58. REGINA. Chris Adam, Jessie Bailey, Martin Bailey, Gaetane Baraby, Laurent Bazin, Margaret Belcher, Tom Beveridge, Frank Brazier, Carol and Lorne Bjorklund, Eric Cooke, Renald Daoust, Bob and Sandra Ewart, Sheallagh Fitzgerald, Greg Kraetzig, Robert Kreba, Wee Lee, Christine MacDonald, John MacDonald, Ron Myers, Mariela Paez, Gillian and Trevor Richardson, Tom Riffel, Janie Wilhelm, Pierre Wilhelm.

59. ROSCOMMON DISTRICT (Kelvington). Chester Sloan, Dianne Sloan, Marguerite Sloan.

60. ROUND LAKE. Doug Francis.

61. ST WALBURG. Nigel Caulkett.

62. SASKATOON. Betty and Bill Albritton, Juhachi, Kango, Mika and Rika Asai, Bob and Joyce Besant, Eveline
Boudreau, Ron Bremner, Donald Carlson, Muriel Carlson, Paul and Vi Coutu, Eric Densem, Anne Dzus, Chris Escott, Colleen Gerwing, Mary Gilliland, Bernard Gollop, John Hanbidge, Ramona Harms, Mary and Stuart Houston, Dick Kerbes, Louise Klaassen, Donna Malcolm, Don and Jo McRobbie, Doris Montalbetti, Dan Neves, Jim and Pat O'Neil, Evelyn Paterson, Don Perkins, John Polson, Vicki Root, Charles Simpson, Jim Smart, Phil Taylor, Bruce, Dale, Doug and Ron Tomlinson, Lena Vella, Lee Wood, Lois Wooding, Kelwin Wylie.

63. SCOTT. Sandra Pederson, Guy Wapple, Robert Wapple.

64. SKULL CREEK. Jim Bennetto, Tim Bennetto, Marjorie Mann, Robert Mann.

65. SOMME. Stan Back, Les Baker, Edwin Billeter, Sharon Birch, David Black, Wesley Black, Florence Chase, Eldon Hooper, Donald Hooper, Margaret Hooper.

66. SPRING VALLEY. Allan Bogdan, Flossie Bogdan, Larry Bogdan, Nick Bogdan, Dean Goian.

67. SQUAW RAPIDS. Wayne Harris, Sheila Lamont, Wayne Renaud, Guy Wapple, Gordon Webber.

68. TISDALE. Kristy Morgan, Susan Morgan.

69. TISDALE. Joyce Mohr.

70. WEYBURN. Leo Belanger, Ray Belanger, Greg Bobbitt, Dick Gutfriend, Cy Marcotte, Nick Postey, Stewart Stairmand, Joe Weisgerber.

71. WHITE BEAR. Sig Jordheim.

72. WHITE BEAR LAKE. D. G. Bobbitt.

73. WHITEBEECH. Ida Wotherspoon, Lindsay Wotherspoon.

74. WOLSELEY SOUTH. Dale Chay, J. Donald Hayward, Frank Rokosh.

75. YORKTON. Bill and Joyce Anaka, Joe Bergerman, Samuel Bergerman, Dale and Paule Hjertaas, Warren Hjertaas, Greg Hodgson, Isabel Lawson, Abbie St. Rose. 


\section{LOCATIONS OF COUNTS}

1. ARDATH-OUTLOOK

2. ASSINIBOIA

3. BANGOR

4. BIGGAR

5. BIG GULLY CREEK

6. BIRCH HILLS

7. BRIGHTWATER RESERVOIR

8. BROADVIEW

9. BROMHEAD

10. CLEMENCEAU

11. CROOKED LAKE

12. DALMENY

13. DILKE

14. DUCK MOUNTAIN

15. DUVAL

16. EASTEND

17. ENDEAVOUR

18. FIR RIVER ROAD

19. FORT QU'APPELLE

20. FORT WALSH

21. GARDINER DAM

22. GLAMIS-WISETON

23. GOODSOIL

24. GOOD SPIRIT LAKE

25. GOVENLOCK
26. GRAND CENTRE-PIERCELAND

27. GUERNSEY

28. HERBERT

29. HUDSON BAY

30. HUMBOLDT

31. INDIAN HEAD

32. KAMSACK

33. KENASTON

34. KINDERSLEY

35. KINDERSLEY

36. KUTAWAGAN LAKE

37. LAKE DIEFENBAKER

38. LA RONGE

39. LAST MOUNTAIN LAKE

40. LEADER

41. LITTLE BEAR LAKE

42. LIVELONG

43. LOON LAKE

44. LOVE-TORCH RIVER

45. LUSELAND

46. MAIDSTONE BRIDGE

47. MARSDEN

48. MELFORT

49. MOOSE JAW

50. NAICAM
51. NIPAWIN

52. NIPAWIN

53. PIKE LAKE

54. PINE CREE REGIONAL PARK

55. PRINCE ALBERT

56. PRINCE ALBERT NATIONAL PARK

57. RAYMORE

58. REGINA

59. ROSCOMMON DISTRICT (Kelvington)

60. ROUND LAKE

61. ST. WALBURC

62. SASKATOON

63. SCOTT

64. SKULL CREEK

65. SOMME

66. SPRING VALLEY

67. SQUAW RAPIDS

68. TISDALE

69. TISDALE

70. WEYBURN

71. WHITE BEAR

72. WHITE BEAR LAKE

73. WHITEBEECH

74. WOLSELEY SOUTH

75. YORKTON

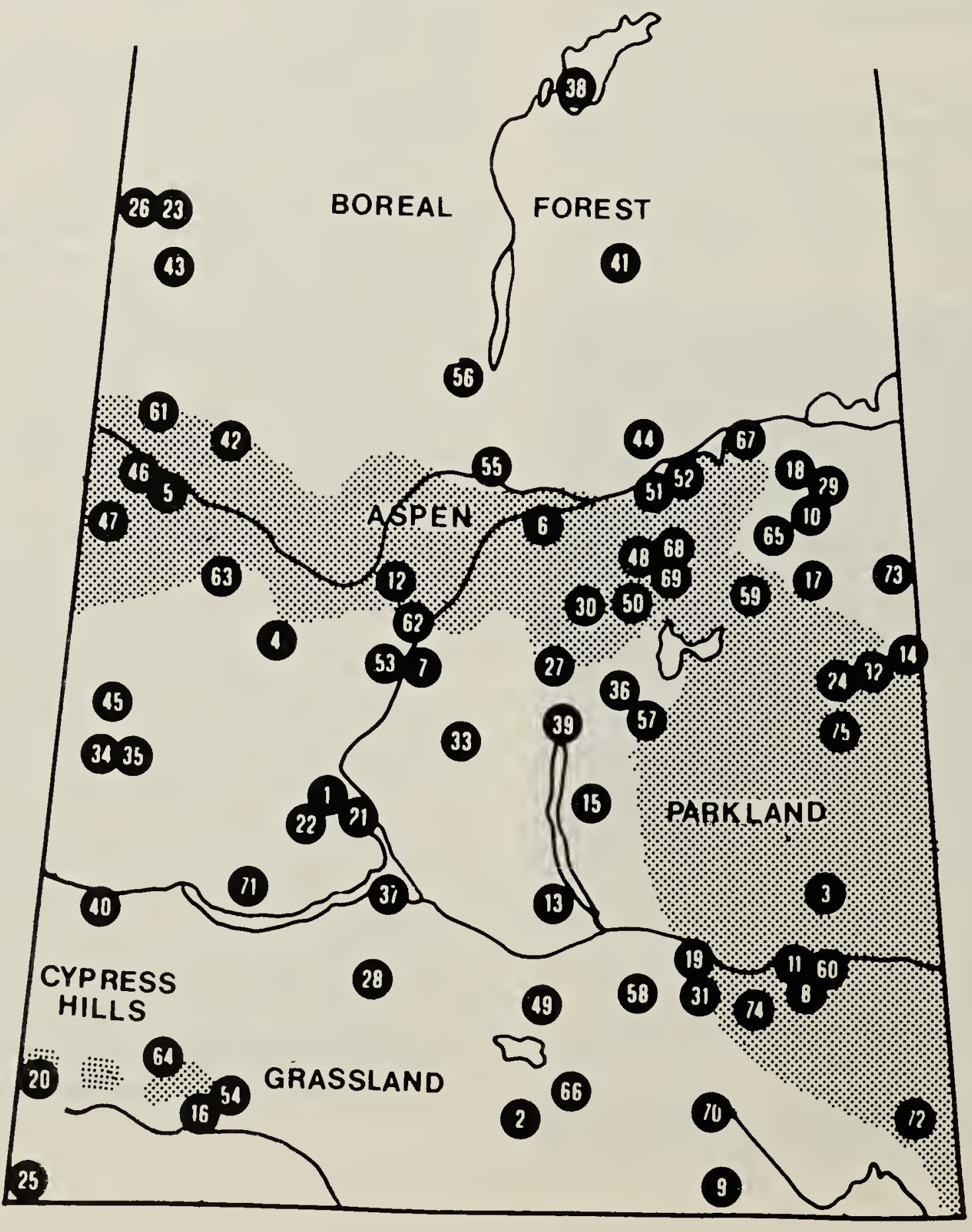


Table 1. COUNT COVERAGE AND WEATHER CONDITIONS

\begin{tabular}{|c|c|c|c|c|c|c|c|c|c|c|c|c|}
\hline 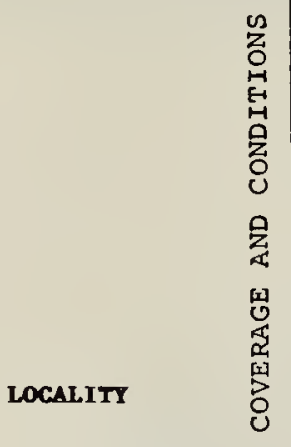 & 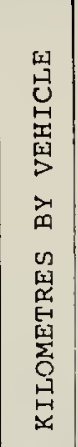 & 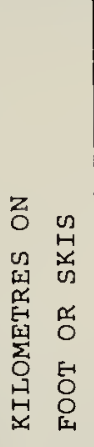 & 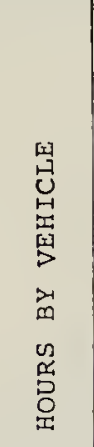 & 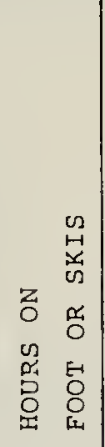 & 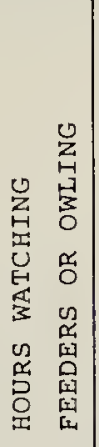 & 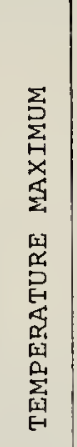 & 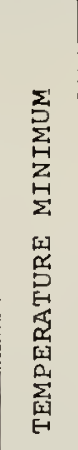 & 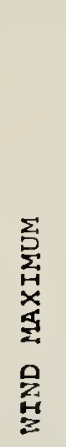 & 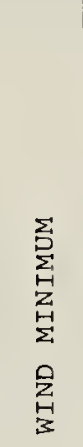 & 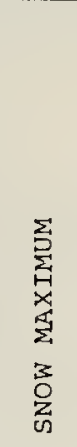 & $\begin{array}{l}\sum_{S} \\
\sum_{n-1} \\
Z \\
Z \\
\Sigma \\
\Sigma \\
z \\
0 \\
z \\
0\end{array}$ & 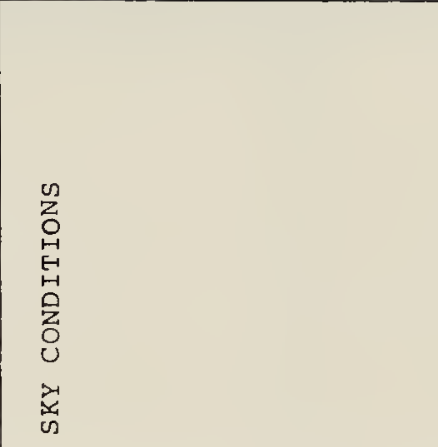 \\
\hline \multirow{5}{*}{$\begin{array}{l}\text { ARDATH-OUTLOOK } \\
\text { ASSINIBOIA } \\
\text { BANGOR } \\
\text { BIGGAR } \\
\text { BIG GULLY CREEK }\end{array}$} & 105 & 5 & 3.5 & 3 & & -10 & -15 & 10 & 0 & 15 & 5 & overcast \\
\hline & 61 & & 2 & & & -4 & -10 & 10 & 5 & 12.5 & & partly cloudy \\
\hline & 250 & & 3.5 & & 2 & -15 & -25 & 40 & 30 & 7 & 5 & overcast, I1ght snow \\
\hline & 187 & 14 & 11.5 & 6 & & -11 & -15 & 40 & 20 & 25 & 0 & cloudy, l1ght 8now \\
\hline & 152 & 19 & 5 & 7 & & -8 & -17 & 48 & 8 & 20 & 5 & clear. \\
\hline \multirow{5}{*}{$\begin{array}{l}\text { BIRCH HILLS } \\
\text { BRIGHTWATER } \\
\text { BROADVIEW } \\
\text { BROMHEAD } \\
\text { CLEMENCEAU }\end{array}$} & 140 & 1.5 & 5.5 & 1 & 1.5 & -15 & -15 & 18 & 13 & 15 & 5 & overcast, clearing \\
\hline & 110 & 2 & 5 & 3 & & -10 & -20 & 15 & 0 & 20 & 0 & cloudy \\
\hline & 87 & 3.5 & 2.5 & 2 & & -6 & -8 & 20 & 15 & 20 & 5 & mostly to partly cloudy \\
\hline & 360 & 2 & 12 & 0.5 & 3 & -6 & -8 & 100 & 60 & 13 & & overcast \\
\hline & 50 & 3 & 1 & 1 & & -20 & -23 & 6 & 4 & 30 & 27 & clear \\
\hline \multirow{5}{*}{$\begin{array}{l}\text { CROOKED LAKE } \\
\text { DALMENY } \\
\text { DILKE } \\
\text { DUCK MOUNTAIN } \\
\text { DUVAL }\end{array}$} & 107 & & 4.5 & & 0.5 & -3 & -2 & 50 & 40 & 20 & 5 & overcast, l1ght snow \\
\hline & & 17.5 & & 6.5 & & -10 & -12 & 40 & 30. & 30 & 0 & partly to mostly cloudy \\
\hline & 134 & 6 & 4 & 2.5 & & -8 & -17 & 15 & 10 & 10 & 0 & partly to mostly cloudy \\
\hline & 109 & 6 & 5 & 3 & & & & 10 & 0 & 35 & 20 & mostly clear,clouding \\
\hline & 288 & 10 & 10 & 3.5 & & -7 & -11 & 17 & 0 & 14 & 0 & partly to mostly cloudy \\
\hline \multirow{5}{*}{$\begin{array}{l}\text { EASTEND } \\
\text { ENDEAVOUR } \\
\text { FIR RIVER ROAD } \\
\text { PORT QU'APPELLE } \\
\text { FORT WALSH }\end{array}$} & 30 & 2 & 3 & 2 & & -3 & -6 & 20 & 5 & 1 & 0 & mostly clear \\
\hline & 5 & 8 & 1 & 6 & & -4 & -18 & 30 & 20 & 29 & 24 & mostly to partly cloudy \\
\hline & 48 & 1 & 2 & 0.5 & & -23 & -25 & 0 & & 35 & 25 & clear \\
\hline & 275 & & 12 & & 6 & 3 & -10 & 40 & 20 & 6 & 4 & overcast \\
\hline & 114 & 18 & 8 & 18.5 & & 2 & -1 & 120 & 10 & 50 & 20 & cloudy, light rain \\
\hline \multirow{5}{*}{$\begin{array}{l}\text { GARDINER DAM } \\
\text { GLAMIS-WISETON } \\
\text { GOODSOIL } \\
\text { GOOD SPIRIT LAKE } \\
\text { GOVENLOCK }\end{array}$} & 300 & 17.5 & 13.5 & 8.5 & & 2 & -8 & 20 & 10 & 40 & 20 & overcast, fog \\
\hline & 45 & 4 & 2.5 & 1.5 & & -5 & -8 & 35 & 5 & 20 & 10 & overcast, flurries, clearing \\
\hline & 75 & & 2 & & 6 & 0 & -2 & 30 & 20 & 12 & 6 & overcast,rain \& snow \\
\hline & 104 & 4 & 5 & 2 & 1 & 2 & 1 & 25 & 15 & 20 & 10 & overcast, 11ght snow \\
\hline & 144 & 26 & 12.5 & 10 & & 2 & 0 & 32 & 3 & 8 & 5 & clear, clouding \\
\hline GRAND CENTRE & 107 & 2 & 4.5 & 2.5 & & -8 & -10 & 11 & 0 & 28 & 20 & overcast \\
\hline GUERNSEY & 6 & & 1 & & & -14 & & 20 & & & & overcast \\
\hline HERBERT & 155 & & 5 & & & -3 & -4 & 12 & 9 & 30 & 10 & partly cloudy \\
\hline HUDSON BAY & & 1 & & 1 & 8 & -20 & -23 & 6 & 4 & 30 & 27 & partly cloudy \\
\hline HUMBOL.DT & 40 & & 3.5 & & 6 & -2 & -5 & 60 & 30 & 10 & 8 & mostly cloudy \\
\hline INDIAN HEAD & 145 & 12 & 9.5 & 3 & 25 & -10 & -12 & 10 & 0 & 10 & 4 & partly cloudy \\
\hline RAMSACK & & & & & & -4 & -9 & 5 & & 30 & 27 & \\
\hline RENASTON & 40 & 1 & 3 & 0.5 & & -8 & -9 & 5 & 0 & 25 & & clear,clouding \\
\hline KINDERSLEY & 10 & & 2 & & & & & & & 20 & 10 & \\
\hline KINDERSLEY & 88 & 2 & 7.5 & 1.5 & & -7 & -9 & 15 & 5 & 16 & 10 & partly cloudy \\
\hline KUTAWAGAN LAKE & 192 & 5 & 8 & 2 & & -7 & -13 & 48 & 16 & 15 & 10 & clear, clouding, clearing \\
\hline LARE DIEFENBAKER & 15 & 3 & 0.5 & 1 & & -7 & -7 & 20 & 15 & 15 & 5 & overcast \\
\hline LA RONGE & 82 & 8 & 5 & & 0.5 & -11 & -15 & 42 & 28 & 30 & 15 & partly cloudy, snow \\
\hline LAST MOUNTAIN LAKE & & & & & & -5 & -15 & 64 & 40 & 15 & 10 & overcast, some snow \\
\hline LEADER & 25 & 4 & 2 & 2 & & -6 & -20 & 4 & 2 & 35 & 25 & mostly cloudy, light snow \\
\hline LITTLE BEAR LAKE & 42 & 1 & 1.5 & 1.5 & & -25 & -30 & 0 & & 30 & 15 & clear \\
\hline LIVELONG & & 5 & & & 9 & 8 & -2 & 0 & & & & clear \\
\hline LOON L.AKE & & & & & 6 & & & & & & & \\
\hline LOVE-TORCH RIVER & 155 & 2 & 7 & 1 & 7 & -10 & -20 & 5 & 0 & 30 & 20 & clear \\
\hline LUSELAND & 118 & 18 & 2 & 5 & 0.5 & -16 & -18 & 30 & 10 & 25 & 5 & overcs8t, l1ght snow \\
\hline MAIDSTONE BRIDGE & 162 & 13 & 8 & 6 & & -8 & -19 & 32 & 16 & 12 & 10 & mostly clear \\
\hline MARSDEN & & & & & 1 & -21 & -23 & 6 & 5 & & & \\
\hline MELFORT & 45 & 2 & 3 & 2 & 2 & -20 & -25 & 20 & 15 & 30 & 20 & clesr \\
\hline MOOSE JAW & 113 & 18 & 5.5 & 16 & 2 & -5 & -7 & 90 & 7 & 25 & 8 & overcast, heavy to $11 \mathrm{ght}$ snow \\
\hline NAICAM & 16 & 4 & 3 & 3 & & -15 & -16 & 40 & 30 & 10 & 2 & most ly cloudy \\
\hline NIPAWIN & 50 & 15 & 4 & 3 & 1 & 0 & -5 & 40 & 30 & 20 & 15 & \\
\hline NIPAWIN & 4 & 2.5 & 0.5 & 1 & 1.5 & -8 & -12 & 10 & & 30 & 20 & overcast, clearing \\
\hline PIKE LAKE & 152 & 38.5 & 16 & 15 & & -9.5 & -21 & 26 & 14.5 & 10 & 0 & partly cloudy \\
\hline PINE CREE PARK & 20 & 6 & 1 & 3.5 & & 5 & 0 & 25 & 20 & 40 & 30 & mostly clear \\
\hline PRINCE ALBERT & 20 & & 0.5 & & 7 & -15 & -20 & 15 & 10 & 30 & 10 & overcast \\
\hline PANP & 145 & 22 & 6.5 & 12 & 1 & -6 & -23 & 8 & 3 & 20 & 15 & overcast, l1ght snow \\
\hline RAYMORE & 264 & 13 & 8 & 4 & $\bar{I}$ & -8 & -16 & 128 & 8 & 25 & 20 & overcast, anow \\
\hline REGINA & 489 & 34.5 & 23.5 & 17 & & 0 & -12 & 35 & 20 & 20 & $7]$ & overcast, 11ght snow \\
\hline ROSCOMON & 7 & & 0.5 & & 8.5 & -5 & & 50 & 40 & 22 & 20 & lovercast hesvy to light snow \\
\hline ROUND LARE & 100 & 2 & 6.5 & 1 & 3 & -12 & -17 & 10 & & 30 & 15 & cloudy,clearing \\
\hline ST WALBURG & 120 & 5 & 5 & 3 & & 0 & -5 & 60 & 30 & 40 & 20 & overcast, light to heavy snow \\
\hline SASRATOON & 567 & 93 & 52 & 50.5 & 0.5 & -1 & -9 & 52 & 22 & 4 & 0 & cloudy, 8now flurries \\
\hline SCOTT & 189 & 7 & 7 & 3.5 & & -10 & -14 & 20 & 0 & 40 & 20 & mostly cloudy \\
\hline SKULL CREER & 20 & 10 & 2 & 4 & & 3 & -2 & 8 & 0 & 50 & 10 & mostly cloudy \\
\hline SOMRE & 193 & 6 & 9 & 2 & 20 & 5 & -2 & 35 & 25 & 30 & 27 & partly cloudy \\
\hline SPRING VALLEY & 78 & 4.5 & 3 & 2 & & -3 & -14 & 8 & & 36 & & overcast, $11 \mathrm{ght}$ now \\
\hline SQDAW RAPIDS & 80 & 38 & 12 & 12 & & -21 & -33 & 40 & 2 & 15 & 10 & partly cloudy \\
\hline TISDALE & & & & & 2 & -18 & & & & & & overcast, moderate snow \\
\hline TISDALE & 30 & & 2 & & 30 & 0 & -20 & 40 & 30 & 12 & 10 & moderate snow \\
\hline MEYBUEW & 265 & 9 & 12 & 4 & 0.5 & 6 & 4 & 7 & 4 & 10 & 6 & partly cloudy, clearing \\
\hline WHITB BRAR & 18 & 6 & 0.5 & 2 & 0.1 & -18 & & 25 & 5 & 32 & 16 & overcast, clearing \\
\hline MHITS BEAR LAKS & 152 & 14 & 4 & 4.5 & & -12 & -20 & 35 & 15 & 15 & 1 & clear,clouding \\
\hline WHITEDEECH & 20 & & 1 & & 6 & -18 & -20 & 5 & 0 & 25 & 20 & clear \\
\hline DOLESLEY SOUTH & 80 & 1 & 2.5 & 0.5 & 0.5 & -8 & -9 & 10 & 5 & 20 & 18 & overcent.11 tht anow \\
\hline roluan & 108 & & 8 & & & -1 & & 25 & 5 & 20 & 10 & overcast \\
\hline
\end{tabular}


Table 2-1. SPECIES RECORDED FROM MORE THAN THREE LOCALITIES.

A plus sign indicates a high count for a species seen during count period but not on count day

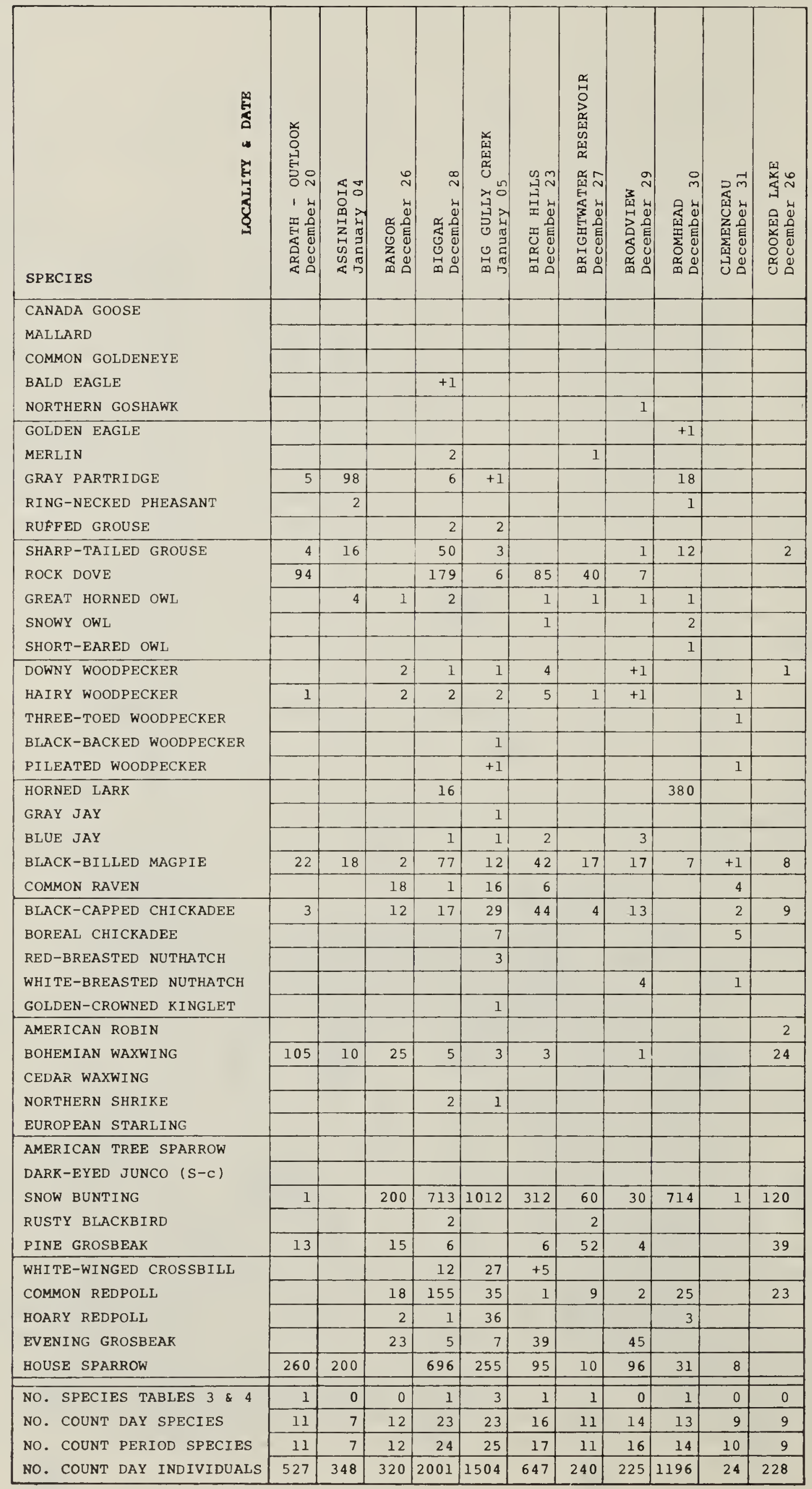


Table 2-2. SPECIES RECORDED FROM MORE THAN THREE LOCALITIES.

A plus sign indicates a high count for a species seen during count period but not on count day

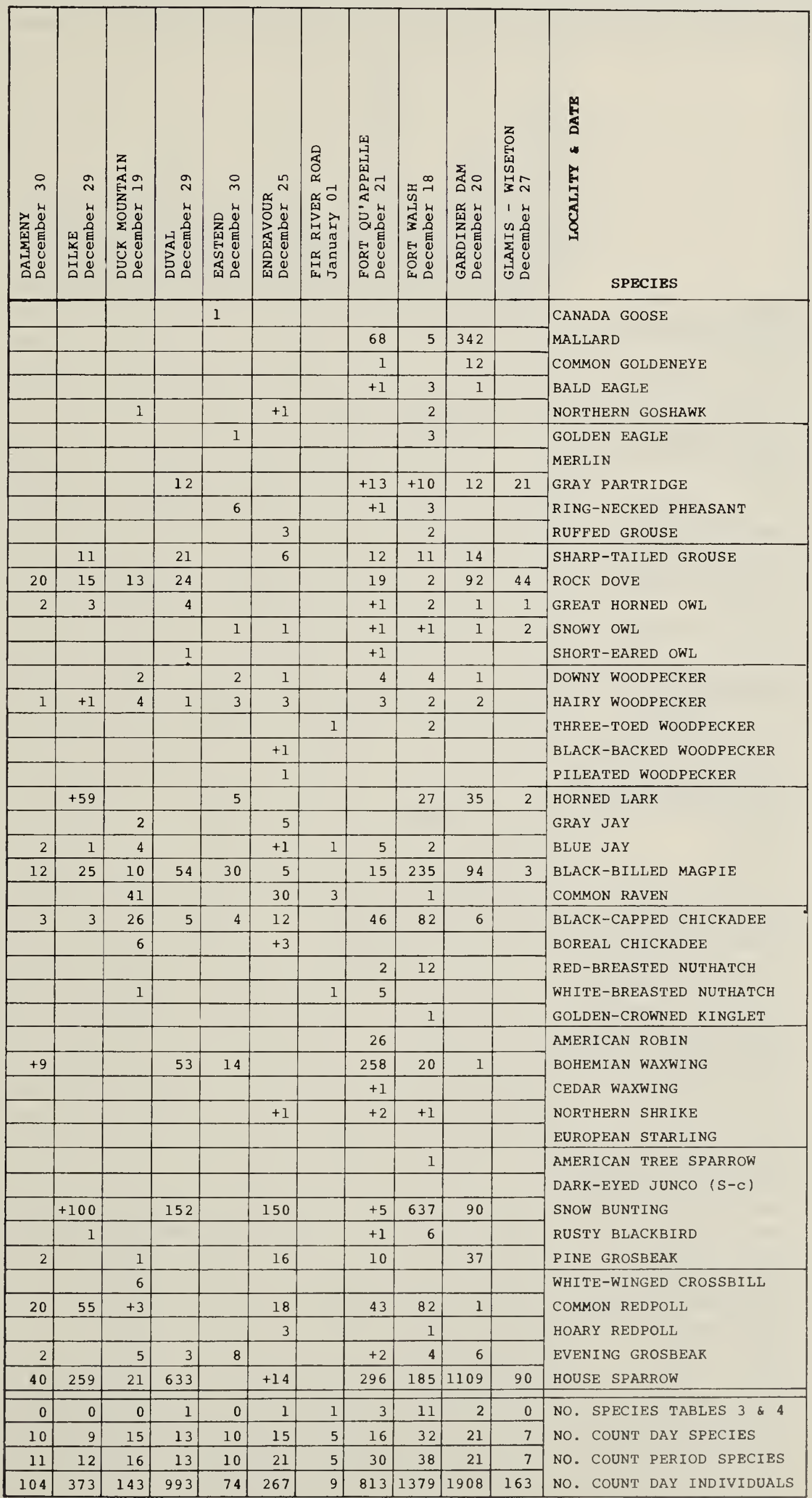


Table 2-3. SPECIES RECORDED FROM MORE THAN THREE LOCALITIES.

A plus sign indicates a high count for a species seen during count period but not on count day

\begin{tabular}{|c|c|c|c|c|c|c|c|c|c|c|c|}
\hline 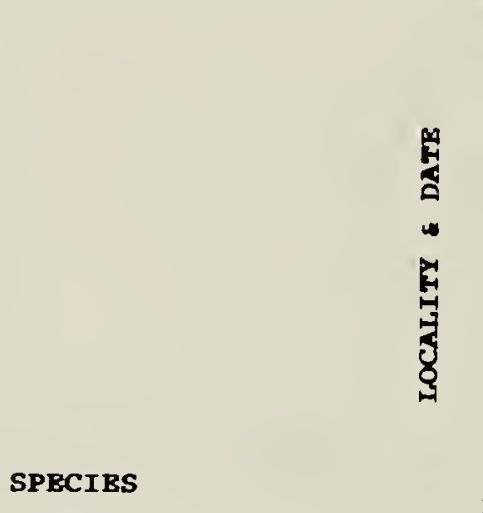 & 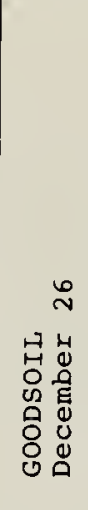 & 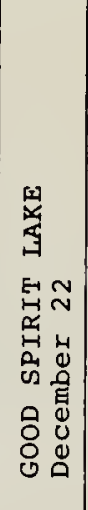 & 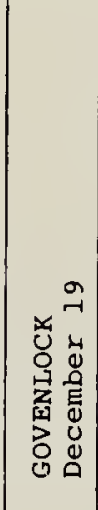 & 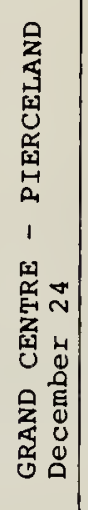 & 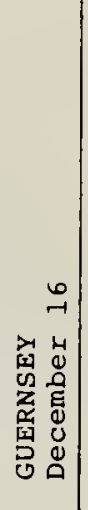 & 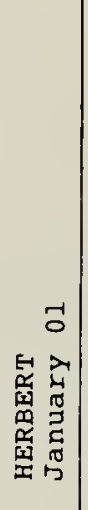 & 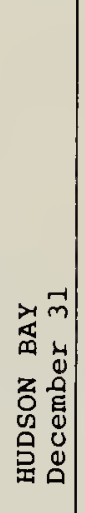 & 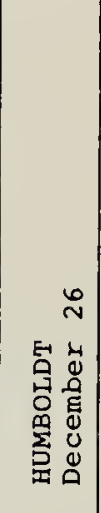 & 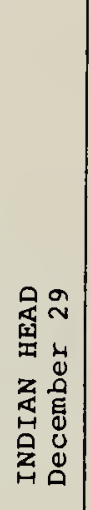 & 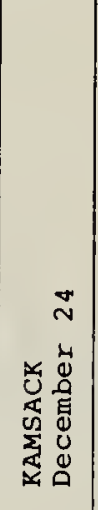 & 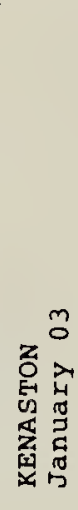 \\
\hline \\
\hline \multirow{4}{*}{$\begin{array}{l}\text { MALLARD } \\
\text { COMMON GOLDENEYE } \\
\text { BALD EAGLE } \\
\text { NORTHERN GOSHAWK }\end{array}$} & & & & & & & & & 35 & & \\
\hline & 7 & & & & & & & & & & \\
\hline & & & & & & & & & 4 & & \\
\hline & & +1 & & 1 & & & & & & & \\
\hline \multirow{5}{*}{$\begin{array}{l}\text { GOLDEN EAGLE } \\
\text { MERLIN } \\
\text { GRAY PARTRIDGE } \\
\text { RING-NECKED PHEASANT } \\
\text { RUFFED GROUSE } \\
\end{array}$} & & & 2 & & & 1 & & & & & +1 \\
\hline & & & & & & 1 & & +1 & & & \\
\hline & & & 59 & & & 99 & +7 & +26 & +12 & 10 & 23 \\
\hline & & & 15 & & & & & & & & \\
\hline & +3 & +1 & & & & & & & 6 & & \\
\hline \multirow{5}{*}{$\begin{array}{l}\text { SHARP-TAILED GROUSE } \\
\text { ROCK DOVE } \\
\text { GREAT HORNED OWL } \\
\text { SNOWY OWL } \\
\text { SHORT-EARED OWL } \\
\end{array}$} & & 12 & 3 & & & 2 & & +5 & 12 & 30 & +1 \\
\hline & & & 3 & 6 & & 6 & & & 63 & & 42 \\
\hline & & +5 & 5 & & & & & 1 & 3 & 1 & \\
\hline & +1 & & 2 & & 1 & 1 & & & 2 & & +1 \\
\hline & & & 2 & & & & & & 1 & & \\
\hline \multirow{5}{*}{$\begin{array}{l}\text { DOWNY WOODPECKER } \\
\text { HAIRY WOODPECKER } \\
\text { THREE-TOED WOODPECKER } \\
\text { BLACK-BACKED WOODPECKER } \\
\text { PILEATED WOODPECKER } \\
\end{array}$} & 2 & 8 & & 1 & & & +1 & 2 & 7 & 2 & \\
\hline & 2 & 9 & & 2 & 1 & & +2 & 2 & 3 & 1 & \\
\hline & & & & & & & & & & & \\
\hline & & & & & & & & & & & \\
\hline & & 1 & & & & & +2 & & & & \\
\hline \multirow{5}{*}{$\begin{array}{l}\text { HORNED LARK } \\
\text { GRAY JAY } \\
\text { BLUE JAY } \\
\text { BLACK-BILLED MAGPIE } \\
\text { COMMON RAVEN } \\
\end{array}$} & & & 3043 & & & 12 & & & 1 & & +1 \\
\hline & 2 & & & 1 & & & 1 & & & & \\
\hline & 4 & +1 & & 8 & & & 10 & +3 & 17 & 4 & 2 \\
\hline & 6 & 21 & 5 & 11 & 3 & 19 & 2 & +1 & 51 & 6 & 12 \\
\hline & 10 & 2 & & 43 & 1 & & 23 & +1 & & 10 & \\
\hline \multirow{5}{*}{$\begin{array}{l}\text { BLACK-CAPPED CHICKADEE } \\
\text { BOREAL CHICKADEE } \\
\text { RED-BREASTED NUTHATCH } \\
\text { WHITE-BREASTED NUTHATCH } \\
\text { GOLDEN-CROWNED KINGLET }\end{array}$} & 6 & 32 & & 17 & & & 17 & 6 & 46 & 23 & +1 \\
\hline & & 1 & & 3 & & & & & +1 & & \\
\hline & & & & & & & & & 16 & & \\
\hline & & 1 & & & & & & & 10 & & \\
\hline & & 2 & & & & & & & & & \\
\hline \multirow{5}{*}{$\begin{array}{l}\text { AMERICAN ROBIN } \\
\text { BOHEMIAN WAXWING } \\
\text { CEDAR WAXWING } \\
\text { NORTHERN SHRIKE } \\
\text { EUROPEAN STARLING }\end{array}$} & & & & & & & & & 5 & & \\
\hline & & 30 & & & & & & +27 & 74 & 140 & \\
\hline & & & & & & & & & & & \\
\hline & & & & & & & & & & 2 & \\
\hline & & & 52 & & 17 & & & & +1 & & \\
\hline \multirow{5}{*}{$\begin{array}{l}\text { AMERICAN TREE SPARROW } \\
\text { DARK-EYED JUNCO (S-C) } \\
\text { SNOW BUNTING } \\
\text { RUSTY BLACKBIRD } \\
\text { PINE GROSBEAK }\end{array}$} & & & 1 & & & & & & & & \\
\hline & 6 & & & & & & & +1 & 1 & 2 & \\
\hline & 100 & 2 & 897 & & 70 & & 80 & +300 & 255 & 150 & 575 \\
\hline & & & 1 & & & & & & 18 & & \\
\hline & +6 & 6 & & 7 & & & 11 & 5 & 28 & 8 & 9 \\
\hline \multirow{5}{*}{$\begin{array}{l}\text { WHITE-WINGED CROSSBILL } \\
\text { COMMON REDPOLL } \\
\text { HOARY REDPOLL } \\
\text { EVENING GROSBEAK } \\
\text { HOUSE SPARROW } \\
\end{array}$} & & & & 45 & & & & & & & \\
\hline & 4 & 117 & 49 & 86 & & +2 & & 35 & 15 & & $\begin{array}{r}+84 \\
\end{array}$ \\
\hline & & 4 & 1 & & & & & & & & \\
\hline & 40 & 1 & & 1 & & & 62 & +1 & 18 & 515 & \\
\hline & 10 & 172 & 1032 & & 50 & 250 & 4 & 5 & 289 & 160 & 125 \\
\hline \multirow{4}{*}{$\begin{array}{l}\text { NO. SPECIES TABLES } 3 \& 4 \\
\text { NO. COUNT DAY SPECIES } \\
\text { NO. COUNT PERIOD SPECIES } \\
\text { NO. COUNT DAY INDIVIDUALS }\end{array}$} & 0 & 0 & 6 & 0 & 0 & 0 & 0 & 1 & 0 & 1 & 0 \\
\hline & 13 & 17 & 22 & 14 & 7 & 9 & 9 & 7 & 25 & 17 & 7 \\
\hline & 16 & 21 & 22 & 14 & 7 & 10 & 13 & 18 & 28 & 17 & 13 \\
\hline & 199 & 421 & 5240 & 232 & 143 & 391 & 210 & 56 & 980 & 1068 & 788 \\
\hline
\end{tabular}


Table 2-4. SPECIES RECORDED FROM MORE THAN THREE LOCALITIES.

A pius sign indicates a high count for a species seen during count period but not on count day

\begin{tabular}{|c|c|c|c|c|c|c|c|c|c|c|c|}
\hline \multirow[t]{8}{*}{ 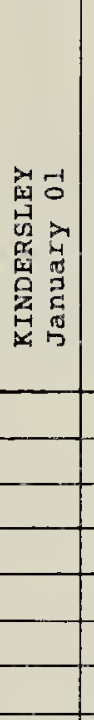 } & 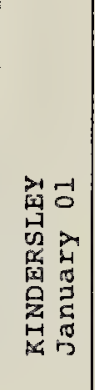 & 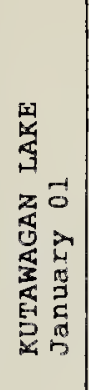 & 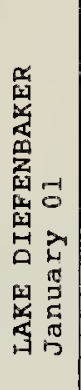 & 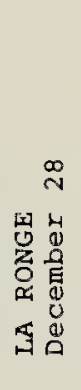 & 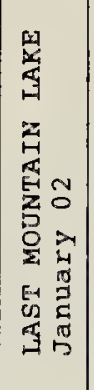 & 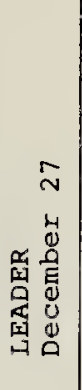 & 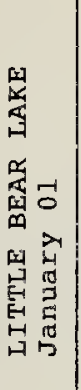 & 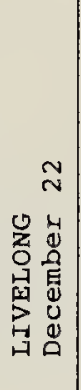 & 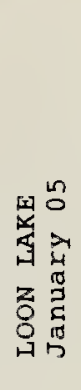 & 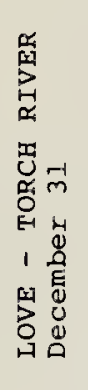 & 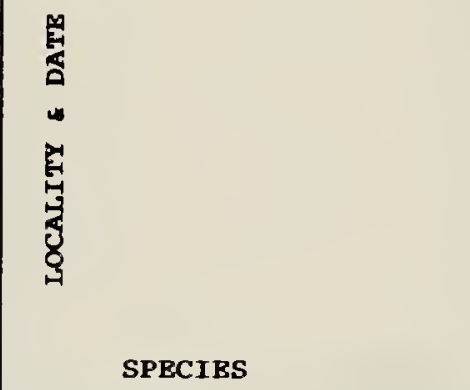 \\
\hline & & & & & & & & & & & \multirow{5}{*}{$\begin{array}{l}\text { CANADA GOOSE } \\
\text { MALLARD } \\
\text { COMMON GOLDENEYE } \\
\text { BALD EAGLE } \\
\text { NORTHERN GOSHAWK } \\
\end{array}$} \\
\hline & & & & & & & & & & & \\
\hline & & & 11 & & & & & & & & \\
\hline & & & & & & & & & & & \\
\hline & & & & & & & & +1 & & 1 & \\
\hline & & 2 & 1 & & +1 & & & & & & \multirow{5}{*}{$\begin{array}{l}\text { GOLDEN EAGLE } \\
\text { MERLIN } \\
\text { GRAY PARTRIDGE } \\
\text { RING-NECKED PHEASANT } \\
\text { RUFFED GROUSE } \\
\end{array}$} \\
\hline & & & & & & & & & & & \\
\hline \multirow[t]{4}{*}{+8} & 4 & 8 & & & 10 & & & & & +13 & \\
\hline & & & & & & 1 & & & & & \\
\hline & & & & & & & & 6 & & 1 & \\
\hline & +5 & 18 & 8 & & 26 & & & & & & \multirow{5}{*}{$\begin{array}{l}\text { SHARP-TAILED GROUSE } \\
\text { ROCK DOVE } \\
\text { GREAT HORNED OWL } \\
\text { SNOWY OWL } \\
\text { SHORT-EARED OWL }\end{array}$} \\
\hline \multirow[t]{2}{*}{4} & & 9 & & & 1 & & & & & & \\
\hline & & 2 & & & 1 & 1 & & & & & \\
\hline \multirow[t]{10}{*}{1} & & & & & +1 & & & +1 & & +1 & \\
\hline & & & & & 2 & & & & & & \\
\hline & & 3 & & 1 & & & & 1 & 2 & 6 & \multirow{5}{*}{$\begin{array}{l}\text { DOWNY WOODPECKER } \\
\text { HAIRY WOODPECKER } \\
\text { THREE-TOED WOODPECKER } \\
\text { BLACK-BACKED WOODPECKER } \\
\text { PILEATED WOODPECKER } \\
\end{array}$} \\
\hline & & & & +2 & & & & 2 & 2 & 5 & \\
\hline & & & & & & & & & & +1 & \\
\hline & & & & & & & & & & & \\
\hline & & & & & & & & & & & \\
\hline & \multirow[t]{3}{*}{5} & & & & +1 & & & & & & \multirow{5}{*}{$\begin{array}{l}\text { HORNED LARK } \\
\text { GRAY JAY } \\
\text { BLUE JAY } \\
\text { BLACK-BILLED MAGPIE } \\
\text { COMMON RAVEN } \\
\end{array}$} \\
\hline & & & & 4 & & & 3 & & 4 & 11 & \\
\hline & & & & 3 & & & & +1 & 5 & 9 & \\
\hline \multirow[t]{8}{*}{3} & 1 & 28 & 3 & & 19 & 28 & & 4 & +3 & 11 & \\
\hline & & & 1 & 67 & & & 3 & 6 & +2 & 42 & \\
\hline & & 2 & & 3 & & 1 & +1 & 12 & 6 & 28 & \multirow{5}{*}{$\begin{array}{l}\text { BLACK-CAPPED CHICKADEE } \\
\text { BOREAL CHICKADEE } \\
\text { RED-BREASTED NUTHATCH } \\
\text { WHITE-BREASTED NUTHATCH } \\
\text { GOLDEN-CROWNED KINGLET }\end{array}$} \\
\hline & & & & +1 & & & +7 & & & & \\
\hline & & & & & & & & +2 & & 2 & \\
\hline & & & & & & & & & & & \\
\hline & & & & & & & & & & & \\
\hline & & & & & & & & & & & \multirow{5}{*}{$\begin{array}{l}\text { AMERICAN ROBIN } \\
\text { BOHEMIAN WAXWING } \\
\text { CEDAR WAXWING } \\
\text { NORTHERN SHRIKE } \\
\text { EUROPEAN STARLING }\end{array}$} \\
\hline \multirow[t]{6}{*}{5} & 3 & & & & & & & +6 & +4 & +25 & \\
\hline & & & & & & & & & & & \\
\hline & & & & & 1 & & & +1 & & & \\
\hline & & & & & 1 & & & & & 5 & \\
\hline & & & & & & & & & & & \multirow{5}{*}{$\begin{array}{l}\text { AMERICAN TREE SPARROW } \\
\text { DARK-EYED JUNCO }(\mathrm{S}-\mathrm{C}) \\
\text { SNOW BUNTING } \\
\text { RUSTY BLACKBIRD } \\
\text { PINE GROSBEAK }\end{array}$} \\
\hline & & & & & & & & & & 1 & \\
\hline \multirow[t]{7}{*}{50} & 17 & 82 & & & 838 & & & 50 & & 98 & \\
\hline & & & & & & & & & & & \\
\hline & & 14 & & 3 & & & & 30 & & 33 & \\
\hline & & & & & & & & & & & \multirow{2}{*}{$\begin{array}{l}\text { WHITE-WINGED CROSSBILL } \\
\text { COMMON REDPOLL }\end{array}$} \\
\hline & & 88 & & 8 & & & & 40 & & 152 & \\
\hline & & +1 & & & & & & & & 8 & \multirow{2}{*}{$\begin{array}{l}\text { HOARY REDPOLL } \\
\text { EVENING GROSBEAK }\end{array}$} \\
\hline & & & & 8 & & & & 50 & 45 & 203 & \\
\hline 50 & +157 & 236 & & & 635 & 40 & & 20 & 60 & 87 & HOUSE SPARROW \\
\hline 0 & 0 & 1 & 1. & 0 & 2. & 0 & 1 & 0 & 0 & 2 & NO. SPECIES TABLES $3 \& 4$ \\
\hline 6 & 5 & 12 & 6 & 8 & 12 & 5 & 3 & 11 & 7 & 20 & NO. COUNT DAY SPECIES \\
\hline 7 & 7 & 13 & 6 & 10 & 15 & 5 & 5 & 17 & 10 & 24 & NO. COUNT PERIOD SPECIES \\
\hline 113 & 30 & 585 & 25 & 97 & 1537 & 71 & 10 & 221 & 124 & 705 & NO. COUNT DAY INDIVIDUALS \\
\hline
\end{tabular}


Table 2-5. SPECIES RECORDED FROM MORE THAN THREE LOCALITIES.

A plus sign indicates a high count for a species seen during count period but not on count day

\begin{tabular}{|c|c|c|c|c|c|c|c|c|c|c|c|}
\hline 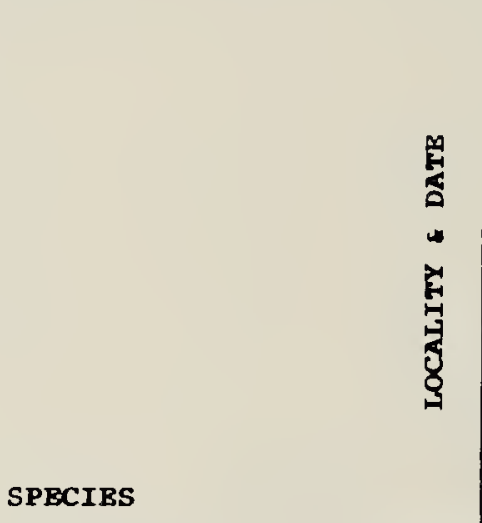 & 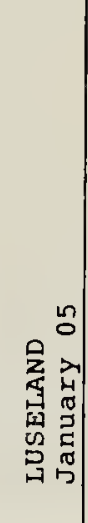 & 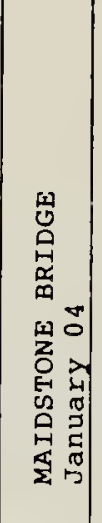 & 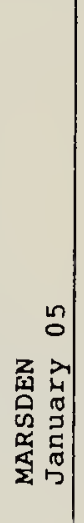 & 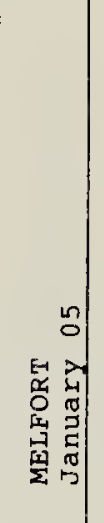 & $\begin{array}{ll} & 0 \\
3 & N \\
3 & 4 \\
5 & 0 \\
0 & 0 \\
5 & E \\
0 & 8 \\
0 & 0 \\
0 & 0\end{array}$ & 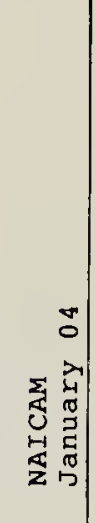 & 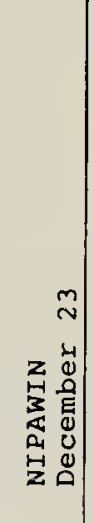 & 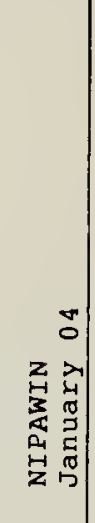 & 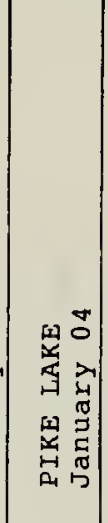 & 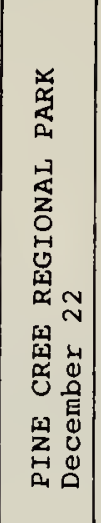 & 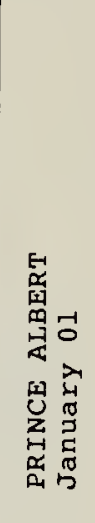 \\
\hline \multirow{5}{*}{$\begin{array}{l}\text { CANADA GOOSE } \\
\text { MALLARD } \\
\text { COMMON GOLDENEYE } \\
\text { BALD EAGLE } \\
\text { NORTHERN GOSHAWK } \\
\end{array}$} & & & & & & & & & +2 & & \\
\hline & & & & & & & 7 & & & & \\
\hline & & & & & & & & & & & \\
\hline & & & & & & & & & & & \\
\hline & & 1 & & & +1 & & & & & 1 & \\
\hline \multirow{5}{*}{$\begin{array}{l}\text { GOLDEN EAGLE } \\
\text { MERLIN } \\
\text { GRAY PARTRIDGE } \\
\text { RING-NECKED PHEASANT } \\
\text { RUFFED GROUSE }\end{array}$} & & 1 & & & 1 & & & & & & \\
\hline & & & & & 2 & & & & & & \\
\hline & 27 & & & & 160 & & & & & 12 & \\
\hline & & & & & 19 & & & & & & \\
\hline & & 1 & & & & & & & 4 & & \\
\hline \multirow{5}{*}{$\begin{array}{l}\text { SHARP-TAILED GROUSE } \\
\text { ROCK DOVE } \\
\text { GREAT HORNED OWL } \\
\text { SNOWY OWL } \\
\text { SHORT-EARED OWL } \\
\end{array}$} & 8 & 12 & & & & & & & 49 & 3 & \\
\hline & 44 & 1 & & & \begin{tabular}{|l|}
459 \\
\end{tabular} & & 130 & & 13 & & \\
\hline & 1 & 1 & & +1 & 1 & & 1 & & & 1 & \\
\hline & 1 & & & +1 & 1 & & 1 & & & & \\
\hline & & & & & & & & & 3 & & \\
\hline \multirow{5}{*}{$\begin{array}{l}\text { DOWNY WOODPECKER } \\
\text { HAIRY WOODPECKER } \\
\text { THREE-TOED WOODPECKER } \\
\text { BLACK-BACKED WOODPECKER } \\
\text { PILEATED WOODPECKER }\end{array}$} & 3 & 2 & 1 & & 2 & +1 & & & 11 & & 1 \\
\hline & & 4 & & +1 & 4 & 2 & & 1 & 11 & & 1 \\
\hline & & & & & & & & & & & \\
\hline & & & & & 1 & & & & & & \\
\hline & & & & & & & & & & & \\
\hline \multirow{5}{*}{$\begin{array}{l}\text { HORNED LARK } \\
\text { GRAY JAY } \\
\text { BLUE JAY } \\
\text { BLACR-BI LLED MAGPIE } \\
\text { COMMON RAVEN } \\
\end{array}$} & 3 & +1 & & & & & & & & 3 & \\
\hline & & & & & & & 2 & & & & \\
\hline & 1 & 3 & & 2 & 1 & & 1 & 2 & 24 & & 1 \\
\hline & 42 & 33 & & 6 & 66 & 13 & 6 & 1 & 131 & 4 & 2 \\
\hline & & 19 & & 1 & & +1 & 43 & 9 & 7 & & 14 \\
\hline \multirow{5}{*}{$\begin{array}{l}\text { BLACR-CAPPED CHICKADEE } \\
\text { BOREAL CHICKADEE } \\
\text { RED-BREASTED NUTHATCH } \\
\text { WHITE-BREASTED NUTHATCH } \\
\text { GOLDEN-CROWNED KINGLET }\end{array}$} & & 15 & 3 & 2 & 26 & 5 & 7 & 8 & 102 & 26 & 12 \\
\hline & & & & & & & & & & & \\
\hline & & & & & 2 & & & & & 18 & \\
\hline & & & & & & & & 1 & & & 1 \\
\hline & & & & & & & 3 & & 1 & & \\
\hline \multirow{5}{*}{$\begin{array}{l}\text { AMERICAN ROBIN } \\
\text { BOHEMIAN WAXWING } \\
\text { CEDAR WAXWING } \\
\text { NORTHERN SHRIKE } \\
\text { EUROPEAN STARLING } \\
\end{array}$} & & & & & & & & & & & \\
\hline & 96 & +3 & & +50 & 30 & +12 & & 7 & 88 & & \\
\hline & 2 & & & & & & & & & & \\
\hline & & 2 & & & & & & & & & \\
\hline & 1 & & & & 7 & & & & 3 & & \\
\hline \multirow{5}{*}{$\begin{array}{l}\text { AMERICAN TREE SPARROW } \\
\text { DARK-EYED JUNCO (S-C) } \\
\text { SNOW BUNTING } \\
\text { RUSTY BLACRBIRD } \\
\text { PINE GROSBEAR }\end{array}$} & & & & & +1 & & & & 1 & & \\
\hline & & & & +15 & 4 & & & & 1 & & \\
\hline & 258 & 2350 & & $\begin{array}{r}+175 \\
\end{array}$ & 4 & 50 & 2 & & 13 & 50 & \\
\hline & & & & & & & & & 1 & & \\
\hline & 2 & 14 & & 18 & 8 & 13 & 14 & 3 & 295 & & \\
\hline \multirow{5}{*}{$\begin{array}{l}\text { WHITE-WINGED CROSSBILL } \\
\text { COMMON REDPOLL } \\
\text { HOARY REDPOLL } \\
\text { EVENING GROSBEAR } \\
\text { HOUSE SPARROW } \\
\end{array}$} & & & & & & & & & & & \\
\hline & 85 & 37 & 3 & +15 & 188 & +8 & 4 & & 450 & 6 & 15 \\
\hline & & 3. & & & & & & & 3 & & 5 \\
\hline & +2 & 7 & & 20 & 17 & & 53 & 52 & 52 & & 50 \\
\hline & 67 & 900 & 2 & 68 & 697 & 12 & 1 & 2 & 90 & & 12 \\
\hline \multirow{4}{*}{$\begin{array}{l}\text { NO. SPECIES TABLES } 3 \& 4 \\
\text { NO. COUNT DAY SPECIES } \\
\text { NO. COUNT PERIOD SPECIES } \\
\text { NO. COUNT DAY INDIVIDUALS }\end{array}$} & 0 & 1 & 1 & 0 & 1 & 0 & 2 & 0 & 1 & 0 & 0 \\
\hline & 16 & 19 & 5 & 7 & 23 & 6 & 15 & 10 & 22 & 10 & 12 \\
\hline & 17 & 21 & 5 & 14 & 25 & 10 & 17 & 10 & 23 & 10 & 12 \\
\hline & 641 & 3491 & 10 & 117 & 1701 & 95 & 275 & 86 & 1524 & 124 & 115 \\
\hline
\end{tabular}


Table 2-6. SPECIES RECORDED FROM MORE THAN THREE LOCALITIES.

A plus sign indicates a high count for a species seen during count period but not on count day

\begin{tabular}{|c|c|c|c|c|c|c|c|c|c|c|c|}
\hline 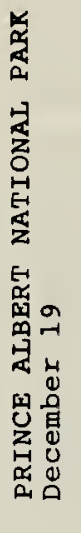 & 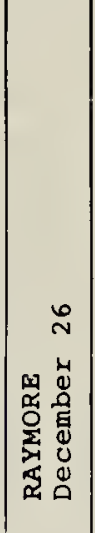 & 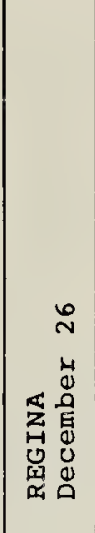 & 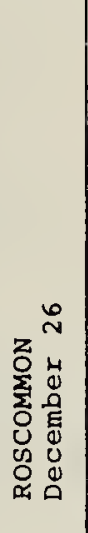 & 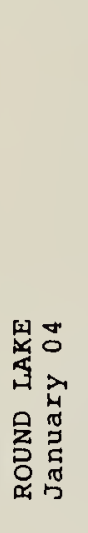 & 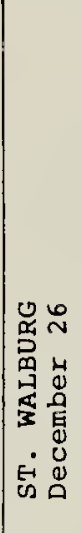 & 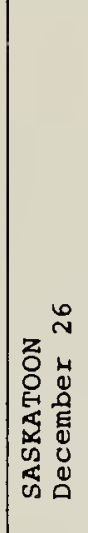 & 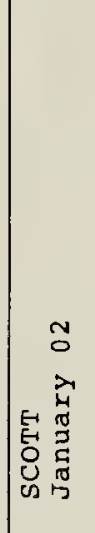 & 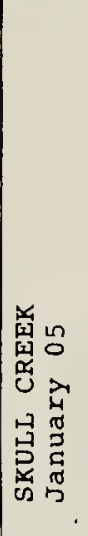 & 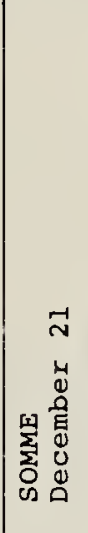 & 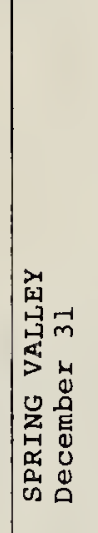 & 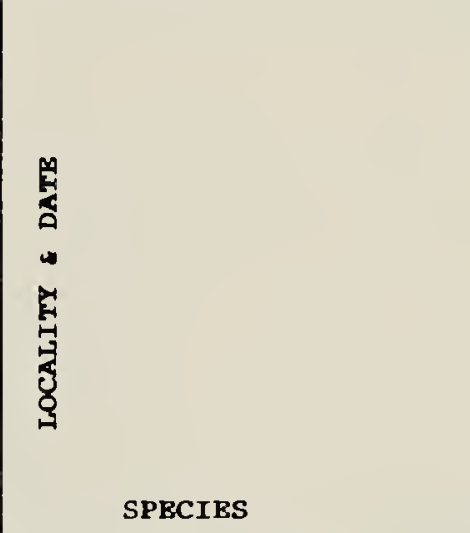 \\
\hline & & 352 & & & & & & 3 & & & CANADA GOOSE \\
\hline \multirow{8}{*}{+1} & & & & 6 & & 36 & & & & & MALLARD \\
\hline & & & & & & 188 & & & & & COMMON GOLDENEYE \\
\hline & +1 & & & & & & & & 1 & & BALD EAGLE \\
\hline & +1 & 1 & & & & +1 & & 2 & +1 & & NORTHERN GOSHAWK \\
\hline & +1 & & & +1 & & & & 1 & & 1 & \multirow{5}{*}{$\begin{array}{l}\text { GOLDEN EAGLE } \\
\text { MERLIN } \\
\text { GRAY PARTRIDGE } \\
\text { RING-NECKED PHEASANT } \\
\text { RUFFED GROUSE }\end{array}$} \\
\hline & 1 & 1 & & & & 9 & & & & & \\
\hline & +7 & 50 & & 13 & & 22 & 39 & +7 & +5 & 94 & \\
\hline & +1 & & & +1 & & & & 7 & & & \\
\hline \multirow[t]{6}{*}{4} & +1 & & & 1 & & 2 & & & 7 & & \\
\hline & 6 & 17 & & 1 & 22 & 58 & & 7 & 21 & 72 & \multirow{5}{*}{$\begin{array}{l}\text { SHARP-TAILED GROUSE } \\
\text { ROCK DOVE } \\
\text { GREAT HORNED OWL } \\
\text { SNOWY OWL } \\
\text { SHORT-EARED OWL }\end{array}$} \\
\hline & 17 & 1248 & & & & 2695 & 21 & & 3 & 38 & \\
\hline & 1 & 3 & & 2 & & 1 & 1 & 2 & +1 & 3 & \\
\hline & 1 & 3 & +1 & & & & 5 & 1 & & 1 & \\
\hline & & & & & & & & 1 & & & \\
\hline 1 & 1 & 3 & 3 & 10 & 1 & 9 & 1 & 2 & 4 & & \multirow{5}{*}{$\begin{array}{l}\text { DOWNY WOODPECKER } \\
\text { HAIRY WOODPECKER } \\
\text { THREE-TOED WOODPECKER } \\
\text { BLACK-BACKED WOODPECKER } \\
\text { PILEATED WOODPECKER }\end{array}$} \\
\hline \multirow[t]{3}{*}{2} & 2 & +1 & 2 & 7 & & 7 & & 1 & 10 & & \\
\hline & & & & & & & & & 1 & & \\
\hline & & +1 & & & & & 1 & & 2 & & \\
\hline \multirow[t]{2}{*}{1} & & & & 1 & & & & & +1 & & \\
\hline & 4 & & & & & & & 54 & +1 & 81 & \multirow{5}{*}{$\begin{array}{l}\text { HORNED LARK } \\
\text { GRAY JAY } \\
\text { BLUE JAY } \\
\text { BLACK-BILLED MAGPIE } \\
\text { COMMON RAVEN }\end{array}$} \\
\hline 19 & & & & & & & & & 12 & & \\
\hline 1 & 3 & & +2 & 7 & & 16 & 3 & 4 & 13 & & \\
\hline 6 & 56 & 29 & 6 & 25 & 3 & 231 & 53 & 7 & 23 & 5 & \\
\hline 27 & +2 & & 2 & & 22 & & & & 63 & & \\
\hline 25 & 11 & 7 & 6 & 20 & 2 & 126 & 2 & 13 & 38 & & \multirow{6}{*}{$\begin{array}{l}\text { BLACK-CAPPED CHICKADEE } \\
\text { BOREAL CHICKADEE } \\
\text { RED-BREASTED NUTHATCH } \\
\text { WHITE-BREASTED NUTHATCH } \\
\text { GOLDEN-CROWNED KINGLET } \\
\text { AMERICAN ROBIN }\end{array}$} \\
\hline 4 & & & & & & & & & 8 & & \\
\hline \multirow[t]{10}{*}{1} & 1 & 11 & & & & 17 & & 1 & & & \\
\hline & & 3 & & 8 & & & & & 2 & & \\
\hline & 1 & & & & & 8 & & & 3 & & \\
\hline & +1 & & & 17 & & 5 & & & & +1 & \\
\hline & 4 & +9 & 35 & 150 & & 2103 & 25 & & 8 & & \multirow{4}{*}{$\begin{array}{l}\text { BOHEMIAN WAXWING } \\
\text { CEDAR WAXWING } \\
\text { NORTHERN SHRIKE } \\
\text { EUROPEAN STARLING } \\
\end{array}$} \\
\hline & 2 & 3 & & & & 1 & & & & & \\
\hline & 1 & +1 & & & & 1 & & & +1 & & \\
\hline & 3 & 7 & & & & 38 & & & & & \\
\hline & & & & +3 & & 2 & & 4 & & & \multirow{5}{*}{$\begin{array}{l}\text { AMERICAN TREE SPARROW } \\
\text { DARK-EYED JUNCO (S-C) } \\
\text { SNOW BUNTING } \\
\text { RUSTY BLACKBIRD } \\
\text { PINE GROSBEAK }\end{array}$} \\
\hline & & 5 & & & & 7 & & & & & \\
\hline \multirow[t]{4}{*}{22} & 30 & 60 & +60 & 75 & 300 & 153 & 154 & 50 & 173 & 453 & \\
\hline & +1 & & & & & & & & & & \\
\hline & 12 & 23 & 9 & 4 & & 179 & 14 & 8 & 9 & & \\
\hline & +8 & 23 & & & & 41 & 20 & & & & \multirow{5}{*}{$\begin{array}{l}\text { WHITE-WINGED CROSSBILL } \\
\text { COMMON REDPOLL } \\
\text { HOARY REDPOLL } \\
\text { EVENING GROSBEAK } \\
\text { HOUSE SPARROW } \\
\end{array}$} \\
\hline \multirow[t]{2}{*}{21} & 30 & 22 & 3 & 100 & 34 & 847 & 12 & & 9 & +18 & \\
\hline & 1 & +1 & & 6 & & 10 & & & 1 & & \\
\hline 16 & 7 & 11 & +8 & 150 & & 25 & 8 & & 86 & & \\
\hline & 1355 & 947 & 2 & 75 & 3 & 1759 & 246 & 10 & 64 & 226 & \\
\hline 2 & 4 & 4 & 0 & 4 & 1 & 5 & 1 & 2 & 1 & 0 & \multirow{4}{*}{$\begin{array}{l}\text { NO. SPECIES TABLES } 3 \& 4 \\
\text { NO. COUNT DAY SPECIES } \\
\text { NO. COUNT PERIOD SPECIES } \\
\text { NO. COUNT DAY INDIVIDUALS }\end{array}$} \\
\hline 16 & 24 & 24 & 9 & 21 & 9 & 32 & 16 & 19 & 23 & 10 & \\
\hline 17 & 36 & 31 & 13 & 27 & 9 & 34 & 16 & 21 & 30 & 12 & \\
\hline 154 & 1578 & 2833 & 68 & 684 & 390 & 8601 & 648 & 177 & 561 & 974 & \\
\hline
\end{tabular}


Table 2-7. SPECIES RECORDED FROM MORE THAN THREE LOCALITIES.

A plus sign indicates a high count for a species seen during count period but not on count day

\begin{tabular}{|c|c|c|c|c|c|c|c|c|c|c|c|c|}
\hline 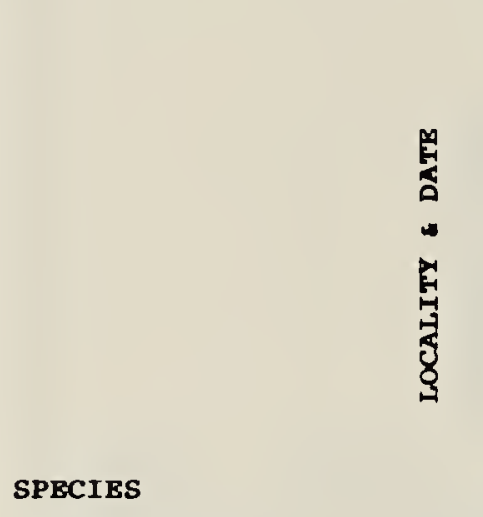 & 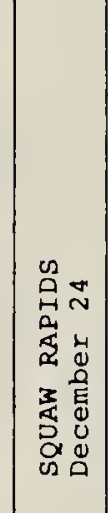 & 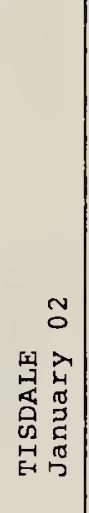 & 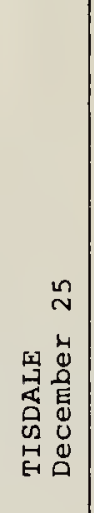 & 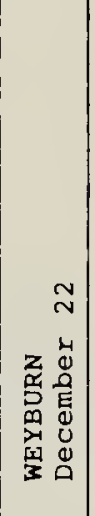 & 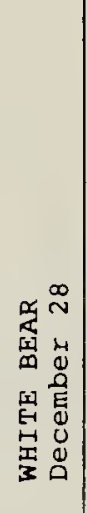 & 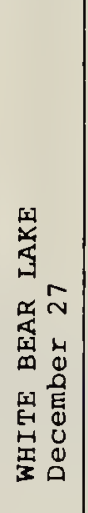 & 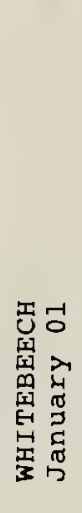 & 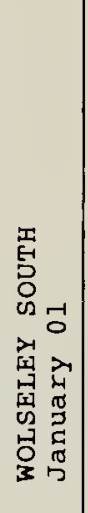 & 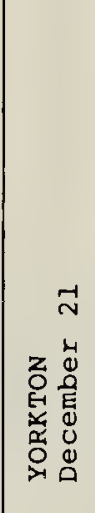 & 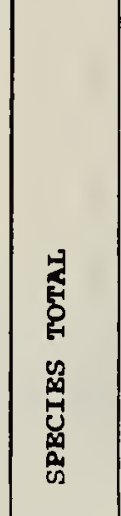 & 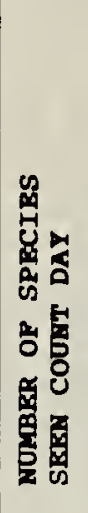 & 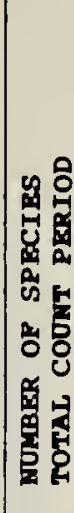 \\
\hline \multirow{5}{*}{$\begin{array}{l}\text { CANADA GOOSE } \\
\text { MALLARD } \\
\text { COMMON GOLDENEYE } \\
\text { BALD EAGLE } \\
\text { NORTHERN GOSHAWK } \\
\end{array}$} & & & & & & & & & & 356 & 3 & 4 \\
\hline & 8 & & & & & & & & & 507 & 8 & 9 \\
\hline & 250 & & & & & & & & & 469 & 6 & 6 \\
\hline & 2 & & & & & & & & & 11 & 5 & 8 \\
\hline & 2 & & & & 1 & & & & & 12 & 10 & 17 \\
\hline \multirow{5}{*}{$\begin{array}{l}\text { GOLDEN EAGLE } \\
\text { MERLIN } \\
\text { GRAY PARTRIDGE } \\
\text { RING-NECKED PHEASANT } \\
\text { RUFFED GROUSE }\end{array}$} & & & & & +2 & & & & & 14 & 10 & 16 \\
\hline & & & & +1 & & & & & & 17 & 7 & 9 \\
\hline & & & & 50 & 3 & & & & 12 & 867 & 25 & 36 \\
\hline & & & & +1 & 4 & & & & & 58 & 9 & 13 \\
\hline & 7 & & & & & & +6 & +1 & & 48 & 14 & 19 \\
\hline \multirow{5}{*}{$\begin{array}{l}\text { SHARP-TAILED GROUSE } \\
\text { ROCK DOVE } \\
\text { GREAT HORNED OWL } \\
\text { SNOWY OWL } \\
\text { SHORT-EARED OWL }\end{array}$} & 3 & & & 16 & 59 & 1 & & 2 & 13 & 644 & 39 & 42 \\
\hline & & & & 122 & 154 & & & 21 & 198 & 5938 & 37 & 37 \\
\hline & 2 & & 1 & 8 & 2 & & & +1 & & 69 & 36 & 41 \\
\hline & & & & & & & & & & 29 & 19 & 28 \\
\hline & & & & & & & & & & 11 & 7 & 8 \\
\hline \multirow{5}{*}{$\begin{array}{l}\text { DOWNY WOODPECKER } \\
\text { HAIRY WOODPECKER } \\
\text { THREE-TOED WOODPECKER } \\
\text { BLACK-BACKED WOODPECKER } \\
\text { PILEATED WOODPECKER }\end{array}$} & 4 & 3 & 4 & 1 & +1 & 1 & 2 & & 1 & 129 & 45 & 49 \\
\hline & 9 & 2 & 4 & 2 & & 4 & 2 & 1 & 1 & 141 & 46 & 52 \\
\hline & 5 & & & & & & & & & 10 & 5 & 6 \\
\hline & 6 & & & & & & & & & 11 & 5 & 7 \\
\hline & 2 & & & & & & & & & 7 & 6 & 9 \\
\hline \multirow{5}{*}{$\begin{array}{l}\text { HORNED LARK } \\
\text { GRAY JAY } \\
\text { BLUE JAY } \\
\text { BLACK-BILLED MAGPIE } \\
\text { COMMON RAVEN }\end{array}$} & & & & 39 & +1 & & & & & 3710 & 16 & 22 \\
\hline & 12 & & & & & & 2 & & & 81 & 15 & 15 \\
\hline & 10 & 1 & & & & 1 & 8 & & 1 & 187 & 39 & 44 \\
\hline & 6 & +1 & 6 & 19 & 47 & 4 & 1 & 6 & 32 & 1807 & 67 & 71 \\
\hline & 75 & & 3 & & & & +4 & & & 613 & 32 & 37 \\
\hline \multirow{5}{*}{$\begin{array}{l}\text { BLACK-CAPPED CHICKADEE } \\
\text { BOREAL CHICKADEE } \\
\text { RED-BREASTED NUTHATCH } \\
\text { WHITE-BREASTED NUTHATCH } \\
\text { GOLDEN-CROWNED KINGLET }\end{array}$} & 34 & 6 & 7 & 3 & +1 & 5 & 10 & 2 & 27 & 1069 & 59 & 62 \\
\hline & 6 & & & & & & & & & 40 & 8 & 12 \\
\hline & 1 & & & +2 & & & & & & 87 & 13 & 15 \\
\hline & 1 & & & & & 2 & & & & 41 & 14 & 14 \\
\hline & 2 & & & & & & & & & 22 & 9 & 9 \\
\hline \multirow{5}{*}{$\begin{array}{l}\text { AMERICAN ROBIN } \\
\text { BOHEMIAN WAXWING } \\
\text { CEDAR WAXWING } \\
\text { NORTHERN SHRIKE } \\
\text { EUROPEAN STARLING }\end{array}$} & & & & & & +1 & & & +1 & 55 & 5 & 9 \\
\hline & 3. & 1 & 10 & +17 & +1 & +49 & & +13 & 325 & 3659 & 32 & 45 \\
\hline & & & & & & & & & & 8 & 4 & 5 \\
\hline & 1 & & & & +1 & & & & & 11 & 8 & 15 \\
\hline & & & & +10 & 1 & & & +10 & & 135 & 11 & 14 \\
\hline \multirow{5}{*}{$\begin{array}{l}\text { AMERICAN TREE SPARROW } \\
\text { DARK-EYED JUNCO (S-C) } \\
\text { SNOW BUNTING } \\
\text { RUSTY BLACKBIRD } \\
\text { PINE GROSBEAK }\end{array}$} & & & & & & & & & & 9 & 5 & 7 \\
\hline & & & 1 & 2 & & & & & & 30 & 10 & 12 \\
\hline & 2312 & & +20 & 50 & 20 & & & 1 & 224 & 14260 & 50 & 56 \\
\hline & & & & +1 & & & & & & 31 & 7 & 10 \\
\hline & 162 & & 4 & 6 & & 6 & +8 & 5 & 6 & 1170 & 46 & 48 \\
\hline \multirow{5}{*}{$\begin{array}{l}\text { WHITE-WINGED CROSSBILL } \\
\text { COMMON REDPOLL } \\
\text { HOARY REDPOLL } \\
\text { EVENING GROSBEAK } \\
\text { HOUSE SPARROW } \\
\end{array}$} & & & & & & & & & & 174 & 7 & 9 \\
\hline & 99 & & 10 & 73 & & 54 & +1 & 45 & 23 & 3241 & 16 & 23 \\
\hline & 2 & & & 3 & & & & & & 93 & 18 & 20 \\
\hline & & 27 & 20 & & & 15 & 40 & 3 & 12 & 1761 & 41 & 45 \\
\hline & & 7 & 10 & 526 & 200 & 24 & +6 & 10 & 406 & 15230 & 61 & 64 \\
\hline \multirow{4}{*}{$\begin{array}{l}\text { NO. SPECIES TABLES } 3 \& 4 \\
\text { NO. COUNT DAY SPECIES } \\
\text { NO. COUNT PERIOD SPECIES } \\
\text { NO. COUNT DAY INDIVIDUALS }\end{array}$} & 7 & 0 & 0 & 1 & 0 & 0 & 0 & 0 & 1 & & & \\
\hline & 34 & 7 & 12 & 15 & 10 & 11 & 7 & 10 & 15 & & & \\
\hline & 34 & 8 & 13 & 22 & 16 & 13 & 12 & 14 & 16 & & & \\
\hline & 3075 & 47 & 80 & 920 & 491 & 117 & 65 & 96 & 1282 & & & \\
\hline
\end{tabular}


Table 3. SPECIES SEEN IN THREE OR FEWER LOCALITIES.

\begin{tabular}{|c|c|}
\hline SPBCIES & LOCATIONS AND NUMBERS SBBN \\
\hline \multirow{5}{*}{$\begin{array}{l}\text { GREAT BLUE HERON } \\
\text { TUNDRA SWAN } \\
\text { TRUMPETER SWAN } \\
\text { NORTHERN PINTAIL } \\
\text { AMERICAN WIGEON } \\
\end{array}$} & FORT WALSH 1 \\
\hline & LAKE DIEFENBAKER 1 \\
\hline & SQUAW RAPIDS 1 \\
\hline & FORT WALSH 1 \\
\hline & ROUND LAAKE + 4 \\
\hline \multirow{5}{*}{$\begin{array}{l}\text { CANVASBACK } \\
\text { REDHEAD } \\
\text { COMMON MERGANSER } \\
\text { SHARP-SHINNED HAWK } \\
\text { COOPER'S HAWK }\end{array}$} & ROUND LAKE + 2 \\
\hline & SASKATOON 1 \\
\hline & GARDINER DAM 35; SQUAW RAPIDS 12 \\
\hline & BIRCH HILLS 1 \\
\hline & RAYMORE +1 \\
\hline \multirow{5}{*}{$\begin{array}{l}\text { ROUGH-LEGGED HAWK } \\
\text { PRAIRIE FALCON } \\
\text { SPRUCE GROUSE } \\
\text { SAGE GROUSE } \\
\text { MOURNING DOVE }\end{array}$} & GOVENLOCK 2; RAYMORE $+1 ;$ SQUAW RAPIDS 1 \\
\hline & BROMHEAD 1; GOVENLOCK 1 ; SKULL CREEK 1 \\
\hline & FIR RIVER 3; LITTLE BEAR LAKE 4; SQUAW RAPIDS 6 \\
\hline & FORT WALSH +3 ; GOVENLOCK 3 \\
\hline & NIPAWIN $(\# 51)+1$ \\
\hline \multirow{5}{*}{$\begin{array}{l}\text { EASTERN SCREECH-OWL } \\
\text { NORTHERN HAWK-OWL } \\
\text { BARRED OWL } \\
\text { GREAT GRAY OWL } \\
\text { BOREAL OWL }\end{array}$} & YORKTON 1 \\
\hline & ROUND LAKE + 2 ; SQUAW RAPIDS 1 \\
\hline & LOVE - TORCH RIVER 1; REGINA + 1 \\
\hline & LOVE - TORCH RIVER 1 ; SQUAW RAPIDS 1 \\
\hline & BIG GULLY CREEK 1 \\
\hline \multirow{5}{*}{$\begin{array}{l}\text { NORTHERN FLICKER } \\
\text { AMERICAN CROW } \\
\text { BROWN CREEPER } \\
\text { TOWNSEND'S SOLITAIRE } \\
\text { FIELD SPARROW }\end{array}$} & HUMBOLDT +1; REGINA +1; SASKATOON 1 \\
\hline & FORT QU'APPELLE +1 \\
\hline & FORT WALSH $1 ;$ P. A. NATIONAL PARK $3 ;$ SASKATOON 3 \\
\hline & REGINA 3 \\
\hline & SASKATOON +1 \\
\hline \multirow{5}{*}{$\begin{array}{l}\text { FOX SPARROW } \\
\text { WHITE-THROATED SPARROW } \\
\text { HARRIS' SAPRROW } \\
\text { LAPLAND LONGSPUR } \\
\text { RED-WINGED BLACKBIRD }\end{array}$} & MOOSE JAW 1 \\
\hline & MARSDEN 1 \\
\hline & FORT QU'APPELLE + 1 \\
\hline & FORT WALSH 1 ; GOVENLOCK $48 ;$ P.A. NATIONAL PARK 1 \\
\hline & GOVENLOCK 6 \\
\hline \multirow{5}{*}{$\begin{array}{l}\text { WESTERN MEADOWLARK } \\
\text { BREWER'S BLACKBIRD } \\
\text { COMMON GRACKLE } \\
\text { ROSY FINCH } \\
\text { PURPLE FINCH } \\
\end{array}$} & FORT WALSH + 1 \\
\hline & SOMME +1; WEYBURN +1 \\
\hline & NIPAWIN (\#51) +1; REGINA 1 \\
\hline & FORT WALSH +5; SKULL CREEK + 50 \\
\hline & FORT QU'APPELLE +1; KAMSACK 4; ROUND LAKE 6 \\
\hline \multirow{2}{*}{$\begin{array}{l}\text { RED CROSSBILL } \\
\text { PINE SISKIN }\end{array}$} & FORT WALSH $20 ;$ RAYMORE $3 ;$ ST. WALBURG 3 \\
\hline & WEYBURN +2 \\
\hline
\end{tabular}

\section{Table 4. BIRDS NOT IDENTIFIED TO SPECIES.}

\begin{tabular}{|c|c|}
\hline SPECIES GROUP & LOCATIONS AND NUMBERS SEEN \\
\hline \multirow{6}{*}{$\begin{array}{l}\text { EAGLE species } \\
\text { ACCIPITER species } \\
\text { FALCON species } \\
\text { CHICKADEE species } \\
\text { FINCH species } \\
\text { CROSSBILL species }\end{array}$} & LAST MOUNTAIN LAKE (1) \\
\hline & FORT WALSH $(1$, small) \\
\hline & SASKATOON (1) \\
\hline & BIG GULLY CREEK (7) \\
\hline & ENDEAVOUR (13), FORT WALSH (9) \\
\hline & SQUAW RAPIDS (8) \\
\hline \multirow[t]{6}{*}{ REDPOLL species } & ARDATH-OUTLOOK (19), BIGGAR (48), BIG GULLY CREEK \\
\hline & (35), BRIGHTWATER RESERVOIR (43), DUVAL (30), \\
\hline & FORT WALSH $(8)$, GARDINER DAM $(15)$, GOVENLOCK $(8)$, \\
\hline & KUTAWAGAN LAKE (93), LAST MOUNTAIN LAKE (2), \\
\hline & MAIDSTONE BRIDGE (85), PIKE LAKE (171), RAYMORE \\
\hline & (25), SCOTT (43), SQUAW RAPIDS (19). \\
\hline
\end{tabular}

